CIENCIA Y SOCIEDAD

Volumen XXV, Número 2

Abril - Junio 2000

HACIA UNA TEORÍA DE REVOLUCIÓN
EL CASO DOMINICANO (I)

Manfred Wilckens

RESUMEN

Con esta monografía tratamos de llenar vacíos empíricos, sobre la historia económica y social de la isla de Santo Domingo/Haití en el siglo XIX, concentrándonos en el lapso de tiempo en que la producción y comercialización del tabaco tenía su importancia destacada para el desarrollo de la isla. Los datos y fechas políticos no están en el centro de nuestra atención, sino su función o papel de red de interdependencias, geográficamente entre Europa y el Caribe, políticamente, entre Estados Nacionales, pero funcionalmente, dentro de un sistema mundial con dos polos heterogéneos opuestos: la Metrópolis y su Periferia.

En este sentido, analizamos la integración de la producción isleña a un mercado parcial, el tabaco dominicano orientado al mercado metropolitano tabaquero, como se manifestaba en su orientación hacia Hamburgo y Bremen. La cristalización de esta cadena de interdependencias surge alrededor de los años veinte del siglo pasado. Se manifiesta empíricamente con la unificación política bajo el mando haitiano. Buscando periodizar esta cadena de interdependencias vamos a demostrar cómo arranca en los años veinte y sufre una modificación profunda y abrupta con el gran incendio de Puerto Plata y Santiago durante la Guerra de Restauración.

PALABRAS CLAVES:

Revolución, mercados, producción tabacalera 


\section{INTRODUCCIÓN GENERAL}

Casi todos los autores dominicanos y extranjeros que trabajaron sobre este tiempo y tema lamentan la falta de material empírico-estadístico. Algunos tuvieron que dejar su plan original de escribir sobre el período de la Primera República, como en el caso de Hoetnik y Gómez. Cuando Luis Gómez concibió su obra Relaciones de Producción dominantes en la sociedad dominicana, $1875 / 1975$ con el "propósito inicial" de arrancar el estudio en el momento de la independencia, tuvo que dejarlo "por la debilidad de su base empírica" en el primer período de 1844-1875.

Hasta este momento se manejan los mercados exteriores siempre como una black-box, sin tener información adecuada para interpretar las interdependencias internas; nadie ha usado hasta ahora, las fuentes alemanas. El mercado comprador se presenta como el "monopolio alemán" y vamos a comprobar, refiriéndonos a una fase de desarrollo, que el mercado alemán perdió más y más fuerza. Tratamos de enseñar en qué forma perdieron las casas comerciales alemanes, después de la Guerra de Restauración, el control sobre el mercado tabacalero dominicano.

Nuestro trabajo va a abrir los archivos alemanes para llenar el vacío empírico sobre los años de la primera República y tratará de verificar y comparar con los datos obtenidos por las investigaciones de los autores Marte y Boin y Serulle, los cuales analizaron los archivos franceses e ingleses.

En el transcurso de nuestra investigación se presentaron obstáculos teóricos que parecieron a primera vista insuperables. Muchos autores, sobre todo de la índole marxista, orientados en unas teorías marxistas ortodoxas, presentan conceptos y modelos altamente abstractos, unos de los miles de "ismos". Nosotros tomamos estos modelos como instrumento heurístico para analizar la realidad presentada en las fuentes.

Fue interesante ver como se desarrolló una argumentación científica alrededor de la producción tabaquera sin tener una base 
sólida empírica usando la cadena argumentativa: monopolioendeudamiento-intercambio desigual, para explicar la miseria y el estancamiento de la República durante el siglo XIX.

Vamos a comprobar la particularidad y singularidad dominicana de un "alto" bienestar social y económico en el período de 1820-1875, que coincide con la larga y próspera fase alemana de un ciclo tipo Kondratieff. El auge que culmina en el despegue germánico de los años 1850-1865, que si se quiere se puede llamar tipo Revolución Industrial. Este ciclo largo, próspero, de más de 45 años de trayectoria tabaquera se parte en dos curvas. La primera, en la que la cantidad de tabaco cultivado sigue creciendo hasta su año culminante, en 1875.

El año 1865, el final de la Guerra de Restauración, coincide con la separación de una coyuntura ascendiente en sus dos componentes: cantidad y calidad de tabaco exportado. La cantidad exportada sigue su ciclo ascendente, pero el precio del producto de este momento en adelante toma una curva descendiente y pierde su estatus de tabaco excelente en calidad. Durante todo el siglo XIX el tabaco dominicano no logra recuperar su viejo esplendor.

Todavía existe cierta falta de un fundamento científicamente sólido de los trabajos que incluyen el tiempo de la Primera República. Un examen crítico de la literatura, preguntando por las fuentes, ideas y/o ideologías que usaron los autores nos presenta un escenario muy heterogéneo.

Lo interesante es que la mayoría de los autores se refieren a. una fuente o mejor a un autor: Juan Bosch, y su obra La composición social dominicana. Este estudio tenía y tiene un impacto tan grande que nadie ha tratado de criticar. A través del estudio de Bosch la segunda fuente es la obra de Hazard: Santo Domingo, Pasado y Presente. Ante los ojos de Hazard se representa la predominancia imaginaria de los comerciantes alemanes en el mercado de tabaco en Puerto Plata. El no es un investigador social crítico y acoge esa apariencia como real. 
Nosotros tratamos de analizar las relaciones vistas por los dos lados: por la Periferia y el Centro. Comenzaremos con la navegación entre Europa y el Nuevo Mundo y su importancia para los dos puertos alemanes: Hamburgo y Bremen, arrancando el análisis antes de que Bremen fuera el centro del mercado internacional del tabaco.

Vamos a discutir la insignificancia del mercado tabaquero dominicano para las dos ciudades hanseaticas. La importancia del comercio alemán.en Puerto Plata no correspondía con el peso en el mercado alemán. El producto dominicano era tratado como cualquier tabaco competitivo de otras regiones. La organización interna del mercado no permitía monopolios. Entonces las dos ciudades no desarrollaron un modelo específico de explotación para el mercado dominicano. Las formas del gran comercio internacional no variaron frente a los diferentes países periféricos.

\section{UN MODELO GENERAL DE REVOLUCIÓN.}

En esta parte seguimos a James C. Davies ${ }^{1}$ en su idea sobre una teoría general de Revolución. Y tratamos de aplicar este modelo a los acontecimientos revolucionarios en la República Dominicana del siglo XIX. Comenzaremos con las ideas de los clásicos sobre el origen de las Revoluciones. Luego, queremos analizar tres revoluciones en la parte dominicana: la Independencia Dominicana, arrancando el día 27 de Febrero de 1844, la Revolución del Cibao en el año 1857, y por fin, la Guerra de Restauración (1863-1865).

Ya Aristóteles creía que la pobreza podría ser una de las causas fundamentales que llevan a un pueblo a la revolución. ${ }^{2}$

Marx y Engels presentaron una teoría de revolución en toda su nitidez o pureza y exigieron que todos los proletarios se unie-

Davies. James C. "Toward a Theory of Revolution", en: American Sociological Review, 27, (1962), pp. 5-19

Aristóteles. Politica, Libro IV. Capitulo XI, traducido y editado al alemán por Nelly Tsouyopoulus y Ernesto Grassi, München. 1965. p. 144 
ran para la Revolución porque no perdían más que sus cadenas. La pauperización creciente lleva a la clase obrera de su resignación hacia la revuelta. Pero esa famosa tesis no es la única de Marx. Él imaginó también otro camino:

"Una mejora en el salario real supone un alto crecimiento del capital productivo. Este crecimiento del capital productivo provoca al mismo tiempo el crecimiento de la riqueza, del lujo, de las aspiraciones sociales y de los placeres. Aun cuando subieran los placeres de los trabajadores, bajarían la posibilidad de satisfacción en comparación con las posibilidades de los capitalistas y del estado de la sociedad en general. Nuestras aspiraciones y placeres nacen en la sociedad, las medimos en la sociedad y no las medimos en los objetos de su satisfacción; porque son de naturaleza social y son de naturaleza relativa", 3

La explicación principal de Marx, a saber, que pauperización creciente hace la revolución, es opuesta a la tesis central en el estudio de Tocqueville sobre la Revolución Francesa. Después de la descripción del desclive económico y social del siglo XVII, y el crecimiento dinámico del siglo XVIII, concluye Tocqueville:

"... asi se quiere decir los franceses encontraron su situación insoportable, que la más se mejoraba [...] No se logra la revolución cuando una situación mala empeora. Muchas veces un pueblo acepta su carga sin lamento, como si no lo sintiera, pero cuando se disminuye la carga estallan violentamente. El gobierno, que se destruye por la revolución es casi siempre mejor que el que la precedió..."

Elegir entre la tesis de Marx y Tocqueville no es fácil. La pregunta es, entonces, ¿cuándo es una revolución probable, en tiempos de prosperidad o en tiempos de depresión?

Nuestro modelo general de revolución, un modelo que trata de explicar cuándo, cómo y por qué estalló una revolución un día dado y está formulada en su hipótesis de base con el siguiente contenido:

\footnotetext{
: Marx y Engels. Obras completas, Tomo VI, Berlin-Este. 1961, p. 397

"Toqueville, Alexis de: El estado viejo y la Revolución [«Der aite Staat und die Revolution»] alemán, Colect. 232, Biersfelden bei Basel, p. 219
} 


\section{Las revoluciones son más probables cuando un perío- do largo de crecimiento económico-social se detiene por una recesión fuerte y abrupta.}

Las dos tesis expuestas, la primera por Marx, la segunda por Tocqueville se pueden interpretar como dos curvas ascendentes relacionadas entre sí, en la que su tensión distancial interna sobrepasa un límite, en la que las fuerzas coherentes internas explotan, en donde los diferentes grupos y clases sociales en juego están en una tensión insoportable.

Las fuerzas dinámicas que conmueven el sistema social establecido, que forman al mismo tiempo su base estructural, mueven la sociedad total hacia un largo crecimiento económico-social. Es, en primer lugar, una curva económica ascendente, pero al mismo tiempo nutrida por una perspectiva interpretativa entre los diferentes grupos sociales que entremezclan sus opiniones sobre una situación real relativa, refiriéndose a un período próspero relativamente largo, comparándolo con un futuro inmediato en peligro de perder todo lo logrado en un momento dado.

En nuestra gráfica la llamamos Curva-ERO. Es la curva de las Esperanzas Reales Obtenidas, una curva bastante elástica por cambios de corto plazo, pero siempre muy cerca de la curva de los ingresos per capita reales, para las grandes masas de la sociedad.

Los movimientos de la Curva-ASP, sobre la curva de base, se nutren más de aspiraciones sociales-políticas, de largo o mediano plazo, bastante inelástica a cambios de corta duración en la superficie de la sociedad. Es más un reflejo de una opinión pública aspirando hacia los cambios sociales-políticos.

Estas dos curvas están, entre sí, estrechamente correlacionadas, como dos curvas psicológicas.

Si la curva-ERO nos indica un movimiento abrupto, brusco hacia abajo, estamos frente a un cambio de prosperidad hacia recesión. El período de prosperidad alimenta la conciencia de 
Gráfica N. ${ }^{\circ} 1$

Teoría General de Revolución

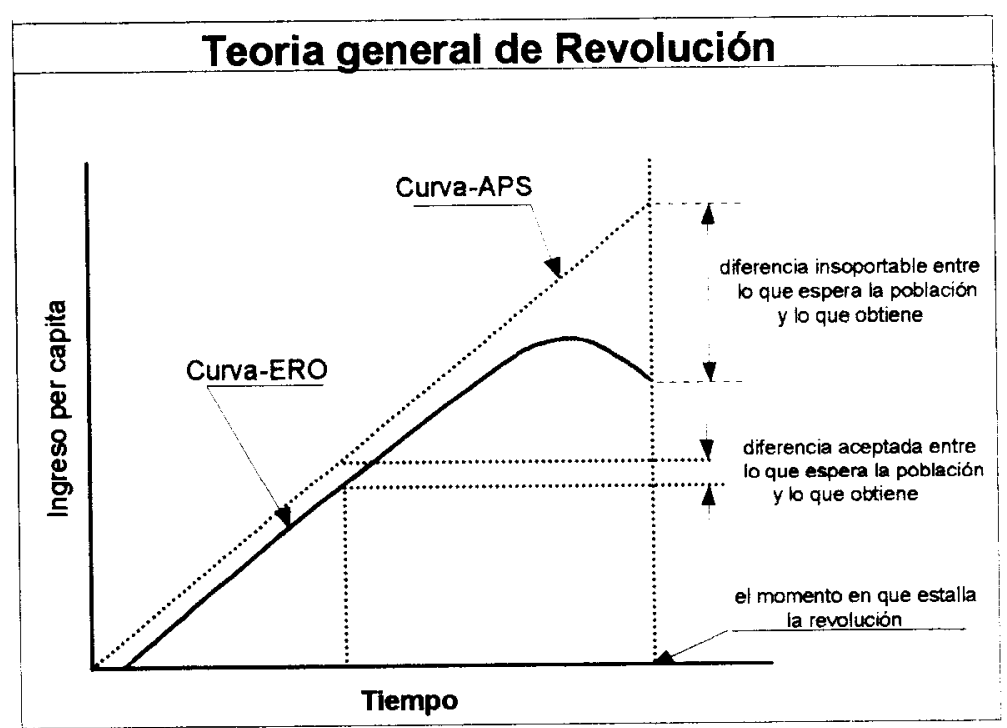

los hombres en una sociedad en la que durante el período de crecimiento sube la expectación de que en el futuro se pueden satisfacer sus aspiraciones crecientes. En la recesión, cuando entre la realidad y las esperanzas optimistas se abre una brecha ancha, se extiende el miedo y la frustración. En este momento, no es importante el nivel real del desarrollo económico y social logrado, sino la esperanza en el progreso, que puede y debe continuar en el futuro. Si aquí se detiene, tenemos la revolución a la puerta.

Estabilidad e inestabilidad política dependen de la conciencia del ambiente general dentro de la sociedad. Gente contenta o apática que no tienen bienes, ni estatus, ni poder, pueden ser políticamente tranquilos; y seres, a los que no les falta nada, estallan. También -y con más probabilidad-se levantan los brazos descontentos mientras que los brazos ricos contentos hacen resistencia a la Revolución. Mas que la falta real de alimentos, es el descontento consciente con las circunstancias, la desigualdad real y la falta de libertad, los que conducen a la Revolución. 
¿Por qué no estallan las revoluciones en una sociedad que está totalmente empobrecida? En este caso extremo, como nos enseñan los estudios de Minesota, y en los KZ-Nazis ${ }^{5}$ los seres humanos se concentran solamente en su instinto de conservación y en ello consumen toda su energía física y mental. El individuo se recoge en sí mismo, se aleja de la sociedad y de cualquier acción que no está directamente relacionada con su supervivencia.

Si los hombres pueden elegir entre perder sus cadenas o su vida, prefieren mantener las cadenas.

\section{LA REVOLUCIÓN RUSA DE 1917}

Vamos a seguir la argumentación de Davies en la aplicación de su teoría en un caso histórico concreto: la Revolución rusa del año 1917.

Con seguridad no podemos decir cuándo comenzó el proceso largo de la modernización del imperio ruso. Los primeros cambios los realizó el zar Pedro el Grande 200 años antes. Y aún cien años antes de la revolución, con la penetración del racionalismo francés a la vida cultural rusa bajo el régimen de Catalina la Grande, el crecimiento de las aspiraciones sociales era invisible.

Seguramente las aspiraciones sociales en el Imperio ruso crecieron durante doscientos años. Un punto importante fue la abolición de servidumbre y la liberación de la masa campesina rusa en el año 1861.

Este ciclo largo de aspiraciones sociales-políticas crecientes logró su punto crítico revolucionario en el año 1905. La densidad de las revueltas violentas campesinas no mermó por la liberación, sino al contrario. Los campesinos lograron la libertad personal, pero no recibieron el factor esencial de su economía: la tierra.

' Cohen. E. A. Human Behavior in the Concentration Camp. New York, 1953, pp. 123-125, 131-140 
Estaban obligados a comprar o alquilar la tierra a un alto costo. Por falta de tierra disponible, se dobló su precio entre los años 1861-1897, con el resultado de que el campesino gastaba gran parte de su ingreso pagando al propietario de la tierra. La libertad personal se convirtió en una servidumbre económica. Incluso en años de buena cosecha permaneció la alimentación de la masa campesina un treinta por ciento por debajo del nivel mínimo saludable.

La presión demográfica empujó la masa rural hacia las ciudades. La ciudad ofrecía, debido a su crecimiento industrial muchos puestos de trabajo a buen salario. Entre los años 1878 y 1897 creció la población en las ciudades en un cien por ciento. La ciudad y la fábrica hicieron crecer nuevas esperanzas y aspiraciones.

Las primeras huelgas de los trabajadores industriales en los años ochenta, fueron aplastadas Estado ruso con toda la violencia posible. Pero estos años, fueron al mismo tiempo el comienzo de una política protectora hacia los trabajadores. El estado prohibió el trabajo infantil y obligaba a los patronos al pago regular de los salarios.

Hay otras pruebas no-económicas de un período con aspiraciones crecientes de la sociedad rusa entre 1861 y 1905. En tiempo de la servidumbre, la nobleza rusa, en general terratenientes, concentraba la justicia en sus manos. Desde 1864 se introdujeron tribunales públicos administrados por jueces preparados. Se redujeron también los castigos corporales. La población estaba entusiasmada por las reformas. Los intelectuales pudieron escribir y pensar con más libertad, criticando incluso a la autocracia zarista.

El Estado ruso llevó a cabo reformas, pero quería mantener sus principios absolutistas. Contra esta invariabilidad de las clases dirigentes creció las critica económica y el radicalismo político. Los radicales asesinaron al zar Alejandro II en 1881. Su 
sucesor trató de limitar las aspiraciones crecientes mediante una política dura contra la población.

Su hijo, Nicolás II, mantuvo las mismas ideas que su padre, pero no tenía la misma vitalidad y dureza. Contra él se formó una oposición formada por intelectuales que apoyaban a los trabajadores y a los campesinos en sus reclamos contra el Emperador ruso. La masa de la población permaneció leal frente al zar, pero quería reformas económicas.

Desde el año 1904 en adelante se formó un "frente de liberación" en el que grupos con ideologías diferentes, desde los liberales hasta los revolucionarios, estaban de acuerdo en que eran necesarias reformas políticas económicas drásticas.

Los acontecimientos de los años 1904-05 marcaron el punto crucial en la curva de las aspiraciones hacia abajo. El Domingo sangriento, el 22 de enero de 1905, marcó el punto de viraje, cuando proletarios hambrientos pero pacíficos pidieron ayuda frente al Palacio de Invierno del zar, y los soldados de su guardia los mataron por centenares. Así terminó el mito del zar como protector de sus súbditos. La respuesta consistió en huelgas por todo el país. Después de las manifestaciones de Octubre, se unieron profesores y estudiantes, al movimiento revolucionario.

El segundo acontecimiento consiste en la derrota catastrófica del ejército y la marina rusa, en la guerra con Japón en 19041905. Esta guerra fue una aventura zarista para expandir el imperio ruso hacia Asia. El pueblo no quería guerra. De los miles de soldados que salieron al frente de la guerra en Asia, regresaron solo unos cientos, heridos y hambrientos. Fue un cuadro deprimente para la población, que vio simbolizada la debilidad del absolutismo del Imperio ruso.

Los años entre 1905 hasta 1917 estuvieron estampados por una miseria creciente. El movimiento de las huelgas decreció en los años de la crisis económica entre los años 1905-1910. En 
plena guerra con Alemania en cambio, aumentaron las huelgas para desembocar en una huelga general en 1917.

La gráfica nos enseña los pequeños altibajos de la curva de aspiraciones y esperanzas. Su primer punto bajo se encuentra en la abolición de la servidumbre; el segundo representa el final del poder del zar. Esta fase larga de 56 años es un período de aspiraciones crecientes provocada por la industrialización que chocaba con una estructura de poder injusta y arbitraria.

La depresión de las esperanzas por el asesinato de Alejandro II en 1881, y la represión siguiente no pudo doblegar la dirección de las aspiraciones sociales y políticas hacia abajo. La segunda depresión resultó por la derrota en la guerra contra Japón. La curva descendiente después que comenzó la guerra con Alemania hace visible el impacto del ataque alemán sobre los sectores ajenos a la producción de guerra. La inflación provocada en 1916 trajo la falta de alimentos y puso a los trabajadores y campesinos en desacuerdo con el gobierno ruso. La tropa misma, mal apro-

Gráfica N. ${ }^{\circ} 2$

El Caso Ruso

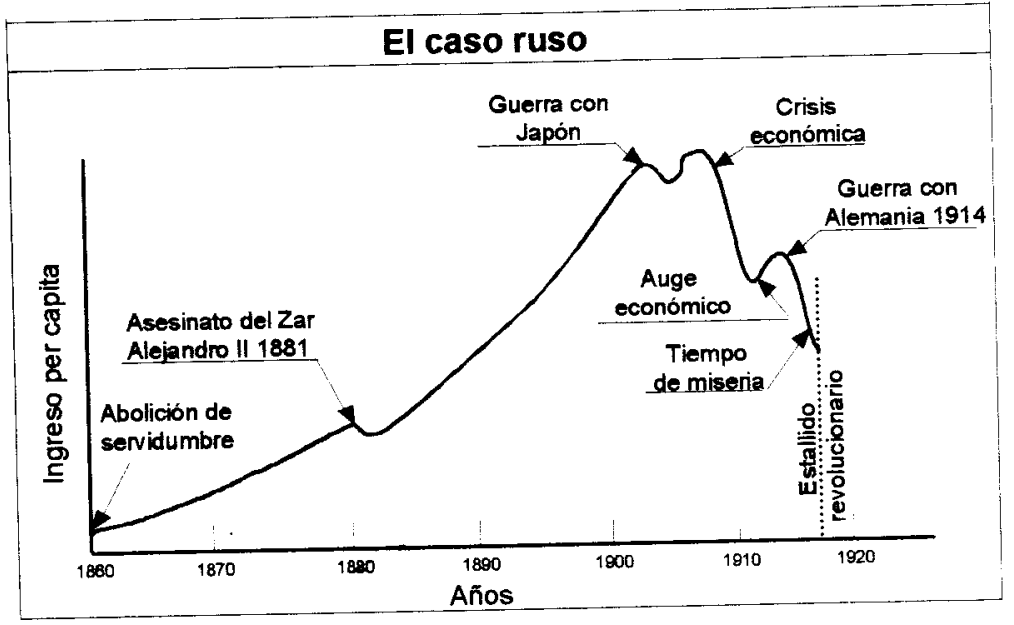


visionada, bajo el mando de superiores brutales y con pérdidas enormes de vidas, hizo crecer la disconformidad en las fuerzas armadas.

El desánimo en la población y en el ejército y la marina alcanzó un punto culminante en la revolución del 17 de febrero 1917 y terminó con la revolución bolchevique del Octubre rojo.

\section{4. ¿QUÉ ES UNA REVOLUCIÓN?}

¡Nada abunda más en los libros que tratan sobre la historia dominicana y en América Latina en general como la palabra Revolución! Cada pequeño motín, revuelta, insurrección o sublevación lleva el nombre de Revolución.

Si hay una definición operacional y empíricamente probada, ¿hasta qué punto se identifica con nuestra Teoría General de Revolución? Las cuatro grandes revoluciones del tiempo moderno se pueden analizar y definir por la teoría de Davies. Los beneficios sociales obtenidos bajaron de una forma drástica antes del estallido de cada revolución. La economía inglesa sufrió una crisis antes del año 1688. Lo mismo pasó en los estados de Nueva Inglaterra, en que la agricultura pasó en 1774-1775 dos cosechas malas en línea. Es casi el mismo escenario en la Francia de los años 1788-1789: después de un largo período de progreso económico, dos cosechas malas. ${ }^{6}$ Rusia en el año 1916-1917 estaba al borde de un colapso económico total, cuando estalló la revolución.

Tocqueville ${ }^{7}$ se acercó al problema de la definición por la vía empírica. Él definió una revolución como la caída de una élite que está legalmente en el poder y que es seguida por un período de cambio social, político y económico intensivo. Brinton ${ }^{8}$ profundizó las ideas de Tocqueville y distingue entre

\footnotetext{
Brinton, Crane. The Anatomy of Revolución, New York. 1952, pp. $31-32$ y 254

7 Toviqueville, Alexis de. Op. cit. p. 7 ss

* Brinton, Crane. Op. cit. pp. 3-4
} 
coup d'état, en que cambia solamente la élite, y las grandes revoluciones.

Lasswell y Kaplan ${ }^{9}$ nos presentan una tipología más precisa. Existen tres tipos de revolución bien marcadas: Revolución de palacio, Revolución política y Revolución social. La revolución de palacio, típica forma de revolución en América-Latina la llamó Lieuwen 10 "caudillismo", usando el término en español.

Los tres tipos de revolución representan diferentes etapas en una escala de intensidad del cambio político-social.

James Rosenau " sigue en este esquema y define la lucha por posiciones (personnel war) dentro de un sistema de dominación ya existente. Un típico ejemplo son los golpe de Estado ocasionados por los caudillos latinoamericanos. La lucha por el sistema de dominación misma (authorithy war) es la lucha de los rebeldes por la ocupación de las posiciones y la lucha por una nueva definición de las posiciones dentro del sistema de dominación. Este tipo está representado por las luchas del poder de sustituir una dictadura por una democracia. La última forma es la lucha por el sistema mismo (structural war) en que los insurrectos tienen como objetivo cambiar la base económica-social de la sociedad. Guerras con la participación de partidos comunistas caen bajo este tipo de revolución.

Samuel Huntington ${ }^{12}$ sigue la misma definición de Rosenau, pero agrega un tipo de revolución intermedia entre revolución de palacio y la toma de poder revolucionario: el Reform coup. El cambio en Argentina en 1955, Venezuela en 1958 y la República Dominicana en 1963 fueron Reform coups.

\footnotetext{
"Lasswel, Harold y Kaplan, Abraham. Power and Society. New Have, Conneticut, 1950, p. $252 \mathrm{~s}$.

1" Lieuwen, Edwin. Arms and Politics in Latin Amerika. New York, 1960, pp. 22-24

"Rosenau, James N. "Internal War as an International Event", en: International Aspects of Civil Strife, editado por James N. Rosenau, Princeton, N. J., 1964, pp. 45-91

12 Huntington, Samuel. "Paterns of Violence in World Politics", en: Channing Patterns of Military Politics, editado por Samuel Huntington. New York, 1962, pp. 17-50
} 
Con esas características mensurables se puede describir los diferentes tipo de revolución? ${ }^{13}$

Tabla N. ${ }^{\circ} 1$

Características de los cuatro tipos de Revolución

\begin{tabular}{|l|c|c|c|c|}
\hline \multicolumn{1}{|c|}{ Tipo } & $\begin{array}{c}\text { Participación } \\
\text { de las } \\
\text { masas }\end{array}$ & Duración & $\begin{array}{c}\text { Violencia } \\
\text { Interna }\end{array}$ & $\begin{array}{c}\text { Objetivos de los } \\
\text { Rebeldes }\end{array}$ \\
\hline $\begin{array}{l}\text { Revolución con } \\
\text { base de masas }\end{array}$ & Fuerte & Larga & Alta & $\begin{array}{c}\text { Cambio de poder y } \\
\text { estructuras }\end{array}$ \\
\hline $\begin{array}{l}\text { Toma de poder } \\
\text { revolucionario }\end{array}$ & Débil & $\begin{array}{c}\text { Corta hasta } \\
\text { mediana }\end{array}$ & $\begin{array}{c}\text { Baja hasta } \\
\text { mediana }\end{array}$ & $\begin{array}{c}\text { Cambio de poder y } \\
\text { estructura limitada }\end{array}$ \\
\hline Reform Coup & Muy débil & Corta & Baja & $\begin{array}{c}\text { Cambio de poder } \\
\text { limitado }\end{array}$ \\
\hline $\begin{array}{l}\text { Revolución de } \\
\text { palacio }\end{array}$ & Ninguna & Muy corta & Ninguna & Ningun cambio \\
\hline
\end{tabular}

5. ANÁLISIS DE LAS TRES REVOLUCIONES DOMINICANAS DEL SIGLO XIX.

\subsection{Los antecedentes de la Independencia Dominicana en 1844.}

Ya habíamos comentado la involucración de la isla Haití/Santo Domingo en un largo período próspero, iniciado por los países europeos en pleno desarrollo hacia la Revolución Industrial. La recuperación económica de las dos partes de la isla comenzó cuando se detuvo el proceso de las inmigraciones fuertes, época en la que el factor esencial de una economía, que casi no usaba una

1 Con la tipología seguimos a: Tanter, Reymond y Midlarsky, Manus. "A Theory of Revolution", en: Journal of Conflict Resolution, 11(1967), pp. 264-280. Editado por la University of Michigan 
tecnología mecanizada e instrumentos que ahorraban trabajo, era la fuerza de trabajo del hombre. Alrededor del año 1818 la Parte española de la isla retomó nuevamente un ritmo acelerado hacia un período largo de prosperidad.

Para la aplicación de nuestro modelo de revolución, está confirmada la existencia de un largo ciclo próspero que hizo crecer el bienestar relativo tanto del pueblo haitiano como del dominicano. Lo que nos interesa ahora es comprobar la existencia de una recesión fuerte y abrupta que se produjo pocos años antes de la fecha memorable del año 1844.

Queremos iniciar esta tarea con el análisis de la literatura especializada sobre el tema. En la mayoría de los trabajos sobre el período de la ocupación haitiana, se encuentra una fuerte corriente anti-haitiana, sobre todo, por parte de los autores dominicanos. La obra más importante sobre este periodo, el trabajo de Frank Moya Pons, La Dominación Haitiana 1822-1844, ${ }^{14}$ como historia política-social, muy orientada hacia los grandes actores del escenario, trata de encontrar respuestas a algunas preguntas de base sobre cuáles fueron las causas de la invasión de Boyer y los factores de su caída y al mismo tiempo cómo fue el desenvolvimiento de la economía en ambas partes. ${ }^{15}$

Moya Pons pone al final de su primer capítulo titulado $L a$ Invasión de Boyer (1820-1822), un resumen de las causas que produjeron la invasión y pusieron fin a la misma. En su dictamen negativo sobre la política económica y social de Boyer, él reduce los múltiples factores a una sola causa:

“... que la situación económica del país había llegado a su punto más bajo de deterioro como resultado de la política económica de Petion y Boyer que al fraccionar y repartir las antiguas plantaciones arruinaron definitivamente la agricultura haitiana y la base de la riqueza de la élite mulata que gobernaba el país. Fue precisamente

${ }_{14}$ Moya Pons, Frank. La Dominación Haitiana 1822-1844. 3" Edición. Santiago, 1978

15 Moya Pons dice en su prefacio: [Él cree que] "... ha logrado contestar esas preguntas. aunque en un nivel de explicación meramente introductorio". Véase: Prefacio a su obra. p. 1 l 
el deterioro económico de Haití acentuado por el peso de la enorme deuda de ciento cincuenta millones de francos impuesta por Francia en 1825 como indemnización de los viejos colonos y como precio del reconocimiento de la independencia haitiana, lo que produjo la caída de Boyer en 1843 y lo que precipitó el movimiento de Separación de los dominicanos que logró consumarse el 27 de Febrero de $1844 " .{ }^{16}$

La argumentación del autor para comprobar su juicio es muy pobre. Las tablas estadísticas que él ofrece al final de su obra no comprueban su tesis de una economía en ruinas. Al parecer, el autor no analizó sus propios datos estadísticos. ${ }^{17} \mathrm{El}$ modelo que nos ofrece Moya Pons se puede reducir: al comienzo y al final ¡una economía en ruinas! Y durante las dos fechas ¿una economía estancada en ruinas? Años mas tarde el autor mantiene el mismo juicio sobre el caso haitiano:

"Con excepción de una agricultura incipiente que fue fomentada con cierto vigor por el gobierno de Boyer, los dominicanos heredaron de los haitianos unas finanzas en desorden, un comercio precario y una industria incipiente". 18

Hay otros autores que son mucho más radicales en su juicio sobre la dominación haitiana. Un autor americano, Otto Schoenrich, escribe después del análisis de la literatura histórica sobre el mismo tema:

Los veintidós años de gobierno haitiano marcaron un período de regresión social y económico para la antigua parte española de la isla. ${ }^{19}$

\footnotetext{
Moya Pons, Frank. La Dominación... Op. cit. pp. 37-38. Este resumen de la política económica de Petión y Boyer fraccionando las grandes plantaciones está en contradicción con lo que escribió Moya Pons sobre la politica de Petión desde el año 1807. Escribe Moya Pons: "Ya desde 1807, Petión habia estado restituyendo las grandes plantaciones de mulatos confiscados en tiempo de Dessalines a sus antiguos dueños". p. 18

i? En diferentes partes de la obra de Moya Pons se encuentran algunos indicadores: para el año 1827 la producción agrícola siguió estancada (p. 72). ¿Y las pruebas? ¿O la crisis mundial de 1825 afectó al mercado de los productos haitianos? (p. 18)

* Moya Pons, Frank. El Pasado Dominicano, Santo Domingo, 1986, p. 156

19 Schoenrich, Otto. Santo Domingo: Un Pais con Futuro. Santo Domingo, 1977, (el original es en inglés, publicado en 1918 en New York), p. 36
} 
¿En que consistió "la regresión social", postulada por el autor de la política de Boyer, "un oscuro mulato"?

"Casi todos los blancos, especialmente las familias más prominentes, los principales representantes del progreso y la cultura de la comunidad, abandonaron definitivamente el país. 20

¿Qué trajo el presidente haitiano al frente de sus tropas a la Parte española? Trajo, como él mismo lo llamó, una "revolución moral". ${ }^{21}$ No le ofrecía él mucho más a una población en su gran mayoría mulata y negra. Una población, según la Constitución española en vigor, separadas en dos clases bien definidas: los ciudadanos y el resto de la población española, "...porque unos son libres, otros libertos y otros ciudadanos". Solamente los últimos podían "obtener empleos municipales y elegir para ellos según los casos", personas que solicitaran y merecieran una Carta de ciudadanía. "Los hombres libres y los libertos, sean pardos, sean morenos, son Españoles, pero no ciudadanos... y los esclavos ni son españoles ni Ciudadanos". ¡Así reza la Constitución española, símbolo del progreso social!

La opinión pública de los dominicanos estaba dividida en tres o más partidos: prohaitiano, procolombiano, prohispano. Boyer venía

“... a voltear de arriba abajo toda esa situación de siglos y que no iba a poder imponerse sino abrumando a los españoles y colombianos; propietarios y comerciantes, con el uso de la fuerza militar". ${ }^{22}$

El presidente haitiano ofreció a la sociedad de la parte española la abolición de la esclavitud y el igualamiento social y jurídico entre blancos, mulatos y negros, y por fin una reforma agraria radical. El quería seguir la misma política de Petión de convertir la mayoría de la población rural en propietarios de un

\footnotetext{
2) Schoenrich, Otto. Santo Domingo Un País ... Op. cit., p. 36

${ }^{21}$ En lo adelante seguimos a Moya Pons: La Dominación ... Op. cit. p. 36

${ }_{22}^{22}$ Ibidem
} 
pedazo de tierra ${ }^{23}$ que les permitiera mantener su familia. Esta política de Petión fue tan exitosa que ayudó a evitar una ocupación por una fuerza extranjera y al mismo tiempo produjo once años de paz (1807-1818).

¡Petión y Boyer no siguieron los consejos sabios de Aristóteles sobre el mismo tema!

Aristóteles ${ }^{24}$ fue el primero que desarrolló una "teoría de la clase media" para una sociedad agraria. En su teoría el mejor fundamento de la sociedad está representado por la mayoría numérica de la propiedad mediana. Es la mejor protección contra cualquier intento revolucionario.

¡Y de este "peligro" emigraron los portadores del progreso y cultura de la sociedad dominicana del siglo XIX! Y ¿a dónde emigraron? ¿A un sistema social avanzado? Schoenrich no lo menciona. Pero Gabriel García nos dice: cuando salió el arzobispo en $1830 \mathrm{y}$ con el "muchas familias y personas respetables" 25 ¡Todos salieron en dirección a Cuba, colonia española, cuya riqueza se basaba en el trabajo forzado del esclavo negro! (sic)

Bosch es el autor que formula las preguntas más adecuadas en materia histórica. En su obra maestra Composición Social Dominicana ${ }^{26}$ escrita al final de los años sesenta, ${ }^{27}$ él especula sobre las causas de la invasión haitiana al territorio de la parte española. El trata de relacionar el acontecimiento político-mili-

23 ¿Los campesinos de hoy no serían muy felices de obtener este mismo "pedazo de tierra" para la manutención de su familia como consecuencia de una reforma agraria moderna?

24 Aristóteles. Política, Libro VI. Capitulo $V$. p. 212. Véase también: Siebek. Hermann. Aristóteles, Stuttgart, p. 113 [en alemán]

25 Gabriel Garcia, José. Compendio de la Historia de Santo Domingo. 5 it edición. Santo Domingo, 1982, Tomo II, p. 144

26 Bosch, Juan. Composición Social Dominicana. Historia e Interpretación, trigésima edición. Santo Domingo 1983

${ }_{27}$ El preámbulo necesario terminado en noviembre 1968 en Benidorm, España. Véase: Bosch, Juan. Op. cit. p. 7 ss. 
tar con una crisis económica, tanto para Santo Domingo como para Haití. ${ }^{28}$

Tal vez eso explicaría -dice Bosch- la actuación de Nuñez de Cáceres al proclamar la independencia dominicana. Y sigue:

"Quizás hubo baja en los precios o pérdidas de cosechas de los productos de exportación, o por lo menos del más importante; en tal caso la invasión del territorio dominicano era una medida que distraía al pueblo haitiano de sus problemas." 29

El autor relaciona los dos acontecimientos políticos en la superficie de la sociedad con una crisis económica en relación con el mercado internacional. Bosch no tiene material empírico a su mano para dar fundamento a sus especulaciones. Pero el presupuesto haitiano bajó un $25 \%$ en comparación con el año anterior (1821). Bosch lo interpreta como "una caída violenta que se produjo en 1822 a causa de desajustes ocurridos en $1821 \ldots$... y pregunta: “¿De qué se trató? ¿Huracanes, sequías, plagas, baja de precios en los mercados compradores extranjeros? No se sabe." 30

Hoy, gracias a los esfuerzos de algunos investigadores, por ejemplo Roberto Marte, ${ }^{31}$ sabemos que no hubo una baja considerable en las exportaciones ni en los precios. La incorporación ${ }^{32}$ de la Parte española produjo, como primer efecto, una subida de las exportaciones de golpe, de $31.7 \%$ a $9,030,397.06$ pesos F. (sic) ${ }^{33}$

\footnotetext{
${ }_{28}$ Bosch, Juan. Op. cit. p. 147 ss.

${ }^{29}$ Ibidem

${ }^{30}$ Ibidem

${ }_{31}$ Marte, Roberto. Estadisticas y Documentos Históricos sobre Santo Domingo (1805-1890). Santo Domingo, 1984 p. 65 ss.

${ }^{32}$ La incorporación se produjo al 9 de febrero de 1822 y la caida del presupuesto del año 1822. no puede servir para explicar la acción politico-militar de Boyer contra la parte española

3 Franklin, James. The Present State of Haiti, London, 1828. pp. 371-372. citado por: Marte. Roberto. Estadisticas ... Op. cit. p. 67
} 
La exportación de café, el producto más importante de Haití bajó un $0.6 \%$, la exportación de algodón subió un $76.7 \%$ (sic), en comparación con el promedio de los años 1818-1821. ${ }^{34}$ Pero si comparamos la exportación del año 1821 con el año anterior, bajó el café un $14.9 \%$ a 299,259 qq. Y el algodón subió un 136\% a 820,563 lbs. El mercado de Inglaterra absorbió más que un tercio de la exportación haitiana en el año $1821 .{ }^{35}$ Los precios del café haitiano en el mercado de Londres bajaron un $16 \%$ en el año 1821 comparado con el año anterior. Pero el mercado del café de Londres pasó por un tiempo muy corto de especulación, en el que los precios subieron de un promedio de 86 chelines por cwt, en los años 1814-1817, a 132 chelines durante los tres años 1818-1820. El precio del café haitiano se fijó en los años 182123 nuevamente a un promedio de 91 chelines por cwt. ${ }^{36}$

Este pequeño movimiento coyuntural de los precios y las rentas hacia abajo, ¿produjo la invasión haitiana al territorio dominicano? Bosch también ve un problema estructural: la falta de tierras para repartir entre los oficiales y los soldados haitianos. Tierra, que faltaba en Haití y abundaba en Santo Domingo. ${ }^{37}$ Bosch lamenta la falta de datos empíricos.

De los pocos datos "... se deduce que el país tuvo algunos años de cierta animación económica, cosa que no se vio en los tiempos de la España Boba, excepto la mejoría en los dos años finales $\ldots{ }^{38}$ El tiempo de la España Boba terminó el 1 Diciembre de 1821 y el día 9 de Febrero del año 1822 incorporó Boyer la Parte española a la República de Haití. ¿Dónde quedó la crisis económica sobre la que especula Bosch, que fue la causa de la acción de Núñez de Cáceres, si estamos frente a una mejoría en los últimos dos años 1820 y 1821 ?

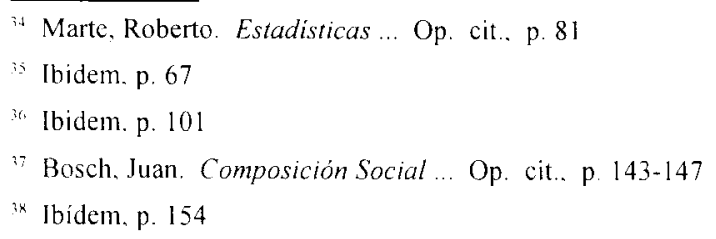


Dejamos por un momento la falta de una crisis al lado, para regresar más tarde a una respuesta posible y probable.

Preocupémonos ahora por los acontecimientos que tuvieron lugar en el año 1838. Para Bosch se formó desde 1822: "Al cabo de algunos años (...) en la llamada Banda Sur una pequeña burguesía de comerciantes, profesionales, dueños de cortes de madera; esa pequeña burguesía se sumó a la de los cultivadores de tabaco y la de los comerciantes del Cibao y acabó tomando en 1838 la dirección de la vida política nacional (...) tomó la dirección en la tarea de organizar a los dominicanos para que lucharan por su independencia". 39

La "cierta animación económica" que concede Bosch a la sociedad en el lapso de tiempo entre 1822 y 1838 , cuando nació la pequeña burguesía, no desembocó " ... en la formación de una burguesía comercial y de ésta habría salido una burguesía industrial, pero en Santo Domingo el comercio no llegó al nivel necesario para eso". ${ }^{40}$

Bosch, implícitamente compara la evolución de la sociedad dominicana con el modelo de un desarrollo "normal", tipo europeo, que por fin termina en un desarrollo industrial.

Pero él sigue en la búsqueda de una crisis, para explicar las causas de la agitación revolucionaria tanto en la Parte española como en la haitiana. ¿Y la respuesta?

"Ahora bien, en medio de esa relativa estabilidad y animación
económica se habia producido algo que ignoramos, algo perturbaba
la vida del país, porque al comenzar el mes de mayo de 1838 se llevó
a cabo en puerto Príncipe un atentado en el cual fue herido de gravedad
un alto funcionario del gobierno, y los autores declararon que su
propósito era derrocar el gobierno de Boyer, y en el mes de julio de
ese mismo año quedaba organizada en Santo Domingo la primera
célula de la Trinitaria. Eso quiere decir que en la primera mitad del
año 1838 toda la isla había entrado en un estado de agitación y

39 Bosch. Juan. Composición Social ... Op. cit.. p. 153

41) Ibidem. p. 157 
conspiración, lo que indica que había malestar, y no podía ser un malestar reciente puesto que las decisiones políticas peligrosas no se toman de golpe y porrazo, de un día para otro; debía tratarse de un malestar que duraba algún tiempo, tal vez dos, cuatro años; quizá más. Para conocer la causa de ese malestar deberíamos estar al tanto de la situación económica en Europa y los Estados Unidos, que compraban los productos de exportación de la Isla. Tal vez hubo baja en los precios de esos productos y eso afectó nuestra economía. De todos modos, se sabe que en 1830 había comenzado en Europa una época revolucionaria y no hay razones para pensar que esa ola no alcanzó de alguna manera a nuestros países de América". ${ }^{41}$

Bosch no tenía datos confiables a mano para detectar la crisis alrededor del año 1838 o algunos años anteriores. Él tenía que especular sobre posibles factores que podían explicar las causas de éstos acontecimientos. Hoy, treinta años más tarde, han aumentado nuestras informaciones estadísticas sobre el comercio de la Isla en este tiempo. Y podemos ver en la Tabla N. ${ }^{\circ} 2$ el desarrollo de la exportación de los productos más importantes de la isla. ${ }^{42}$

Tabla $\mathbf{N}^{\circ} 2$

\begin{tabular}{|c|c|r|r|r|}
\hline Años & Café & Algodón & \multicolumn{1}{c|}{ Tabaco } & \multicolumn{1}{c|}{ Caoba } \\
\hline 1822 & $24,235,372$ & 592,368 & 588,957 & $2,622,277$ \\
1823 & $33,802,837$ & 332,256 & 387,014 & $2,369,047$ \\
1824 & $44,269,084$ & $1,028,045$ & 718,679 & $2,181,747$ \\
1825 & $36,034,300$ & 815,697 & 503,425 & $2,986,469$ \\
1826 & $32,189,784$ & 620,972 & 340,588 & $2,136,984$ \\
1835 & $48,352,371$ & $1,649,717$ & $2,086,606$ & $5,413,316$ \\
1836 & $37,662,672$ & $1,072,555$ & $1,222,716$ & $4,954,944$ \\
1837 & $30,845,400$ & $1,013,171$ & $2,191,874 *$ & $4,789,262$ \\
1838 & $49,820,241$ & $1,285,663 *$ & $2,310,288 *$ & $4,880,873 *$ \\
\hline
\end{tabular}

4 Bosch, Juan. Composición Social ... Op. cit. , p. 157

${ }^{42}$ Marte, Roberto. Estadisticas ... Op. cit., p. 81. Las cifras con $\left({ }^{*}\right)$ las obtuvimos de: Delitsch, Otto Dr. Oesteindia y los paises de la Antártica, Leipzig. 1871, p. 1955. 
La interpretación de los datos estadísticos que tenemos a mano, que Bosch no tenía, nos enseña un panorama muy diferente. No encontramos un malestar, ninguna influencia de una crisis económica que bajó la cantidad de las exportaciones de los principales productos haitianos. Al contrario, esta "cierta animación económica y relativa estabilidad" tenía una dinámica interna que subió la exportación haitiana a niveles no conocidos. Estas cifras nos presentan un "milagro económico", pues a pesar de las vicisitudes climáticas, los movimientos sociales, y la competencia mundial en los mercados europeos, sigue la producción en una curva ascendente.

Tabla N.$^{\circ} 3$

Exportación promedio de Haití durante los períodos 1822-1826, 1835-1838, 1839-1843

\begin{tabular}{|c|c|r|r|r|}
\hline Período & \multicolumn{1}{c|}{ Café } & \multicolumn{1}{c|}{ Algodón } & \multicolumn{1}{c|}{ Tabaco } & \multicolumn{1}{c|}{ Caoba } \\
\hline $1822-1826$ & $34,106,282$ & 677,868 & 507,733 & $2,459,305$ \\
$1835-1838$ & $41,167.171$ & $1,255,277$ & $1,952,871$ & $5,009,599$ \\
$1839-1843$ & $40,763,818$ & $1,095,677$ & $2,236,459$ & $5,041,658$ \\
\hline
\end{tabular}

Tabla N. ${ }^{\circ} 4$

Crecimiento porcentual de exportación de Haití en los períodos 1822-1826 y 1839-1843

\begin{tabular}{|l|r|}
\hline Productos & En \% \\
\hline Café & 19.5 \\
Algodón & 61.6 \\
Tabaco & 340.5 \\
Caoba & 105.0 \\
\hline
\end{tabular}


En este mismo período de la unificación de las dos partes de la isla explotó el comercio del Palo de tinte, más tarde llamado Campeche. De un nivel bastante bajo de exportación promedia de 5,603 toneladas subió a 31,084 toneladas entre 1822-1826 y 1839-1843, una subida de $454.7 \%$ (sic).

Tabla N. ${ }^{\circ} 5$

\section{Crecimiento porcentual de exportación de Haiti en los períodos $1835-1838$ y 1839-1843}

\begin{tabular}{|l|r|}
\hline Productos & En \% \\
\hline Café & -2.2 \\
Algodón & -12.8 \\
Tabaco & +14.5 \\
Caoba & +0.6 \\
\hline
\end{tabular}

Como vemos, se puede interpretar que el crecimiento estaba moviéndose a una curva saturada de alto nivel. Algunos autores quieren interpretarla como una economía estancada. ${ }^{43}$

Se puede especular si este éxito de Haití fue el resultado de una buena política económica por Boyer o al revés a pesar de su mala política económica. Los actores en economías altamente liberadas, en que el "estado" no está presente o en una forma muy reducida, son los campesinos mismos, en colaboración con el sector comercial local e internacional que empuja la producción hacia arriba. Pensamos, que estamos frente a un impacto positivo del crecimiento comercial secular del Moderno Sistema Mundial en que la isla caribeña participó, como reflejo de un crecimiento explosivo de la demanda europea y americana hacia los productos tropicales. Al mismo tiempo fue una prueba de la

\footnotetext{
4: Moya Pons. Frank. La Dominación ... Op. cit
} 
integración de la isla en la distribución internacional de trabajo, hoy llamada Globalización.

La sociedad isleña respondía, como otros sistemas culturales, con un crecimiento extraordinario de la clase de agentes sociales que estaban involucrados en la comercialización de los productos tanto de exportación como de importación. Por supuesto fue un crecimiento de todo el sector comercial y el sector urbano a costo de las masas rurales analfabetas que no tenían acceso a las informaciones de mercado sobre los precios en boga. Dice Lepelletier de Saint Remy:

"En este país, nadie es comerciante, sino por el contrario todo el mundo mercadea. Como nadie tiene con qué vivir, todos tratan de ganarse la vida vendiendo. Militares, abogados, diputados, senadores, administradores, propietarios, por sí mismos o por mujeres, tienen tienda abierta y esa inmensa concurrencia no hace sino aumentar el malestar universal, sin dejar beneficio a nadie". ${ }^{44}$

El autor de la Composición Social Dominicana nos deja un hueco en su explicación sobre las causas que condujeron a la independencia dominicana. La llamada revolución de la Reforma se desarrolló más en la parte haitiana. Bosch no nos ofrece una crisis económica en la parte haitiana ni dominicana. Lo que nos ofrece es: un proceso de concientización de los actores en el escenario de las acciones revolucionarias, que llevaron a la parte dominicana a la separación de la República de Haití, el 27 de Febrero 1844.

La separación fue el resultado de un desarrollo interno de un grupo social llamado pequeña burguesía dominicana que "... tomó conciencia de su naciente autoridad política y social". "

Estamos de acuerdo con Bosch, creemos en la conciencia de su propio valor político y social, pero, ¿nacido de qué o de quién?

\footnotetext{
${ }^{44}$ Lepelletier de Saint Remy, R. Santo Domingo Estudio y solución nueva de la cuestión haitiana, Tomo II, Santo Domingo, 1978, p. 139. El original en francés fue publicado en París en el año 1846.

45 Bosch, Juan. Composición ... Op. cit., p. 158
} 
¿Dónde está la madre engendradora? ¡Bosch no dio una explicación racional de este fenómeno! Él no captó la singularidad de un período largo de prosperidad.

Bosch creó el mito del " ...estado de miseria [que] era consustancial con la existencia misma de la sociedad". ${ }^{40}$

Este mito estorbó la caracterización correcta del período de la Independencia y la revolución en 1857.

En definitiva, buscó el malestar del año 1838 o los años anteriores guiado por su propia teoría, pero jamás pudo encontrarlo porque no existía!

Examinemos la coyuntura de los dos años 1837 y 1838 en que creció visiblemente la conciencia de la pequeña burguesía urbana y no solamente la que tenía sus manos el negocio del tabaco, sino toda la Parte española movida por efectos multiplicadores hacia un bienestar no conocido. La ciudad de Hamburgo aumentó sus compras de la hoja preciosa en el Cibao de 871 quintales métricos a 3,686 quintales una subida de $351 \%$ $\mathrm{y}$ el ingreso en dinero fuerte ascendió de un golpe en $493.9 \%$ (sic) Este era el "malestar" que andaba buscando Bosch.

Regresamos a la conciencia creciente del sector comercial el cual aumentó sus ganancias de forma exorbitante. Marx nos dice:

"Nuestras aspiraciones y placeres nacen en la sociedad, las medimos en la sociedad $[\ldots]$ son de naturaleza social y son de naturaleza relativa". ${ }^{47}$

Vamos a medir la naturaleza relativa de éste crecimiento extraordinario de la producción y consumo dentro de la perspectiva de una generación, una generación bastante contenta de la masa de la población, incluyendo tanto a la clase media rural como a la urbana. Un largo período de paz y acumulación de ciertos ahorros y un bienestar muy alto, dentro del modelo económico so-

\footnotetext{
${ }^{46}$ Bosch, Juan. Guerra de la Restauración, Santo Domingo. 1991. $7^{\text {ma }}$ Edición, Op. cit., p. 20

17 Marx. Engels, ... Op. cit., Tomo VI, Berlin-Este. 1961. p. 397
} 
cial haitiano/dominicano. Un estado general de prosperidad medido en altos índices: un crecimiento fuerte de la población rural y urbana y, a pesar del alto crecimiento poblacional, un estado de pleno empleo, altos salarios y buena salud.

Bosch escribe para lectores de los años setenta de este siglo y apunta:

"Miseria en esa época significaba vivir en un nivel tan bajo que los dominicanos de hoy no pueden ni siquiera imaginárselo. ${ }^{48}$

Nuestra posición es antitética: ningún dominicano de hoy que vive en una casucha de cartón y lata, que vende frutas en los cruces de las calles y avenidas por donde andan muchos Mercedes Benz y Jeepetas, puede imaginarse qué bien vivian sus antepasados en los pueblos y campos en los tiempos de Haití y de la Independencia.

\subsection{LA CURVA-ERO ANTES dEL 27 DE FEbRERO dE 1844.}

Nuestra curva-ERO, una curva de las esperanzas reales obtenidas es en primer lugar la interpretación de una esperanza a corto plazo nutrida por los ingresos obtenidos per cápita del pasado reciente y su proyección al futuro inmediato. Por supuesto, la situación económica de las diferentes clases sociales es un agregado de muchas curvas individuales. Nuestra curva-ERO es una abstracción de mieles de individuos que opinan sobre sus pretensiones inmediatas. Bajo ciertas circunstancias reacciona la curva-ERO bruscamente hacia abajo, forzada por un acontecimiento que presenta el futuro bastante oscuro sin esperanzas frente a una situación económica objetiva.

Comencemos con el estado económico del sector más dinámico de la parte española: producción y exportación del tabaco dominicano. Este nos muestra un escenario alegre y positivo en

${ }_{48}$ Bosch, Juan. Guerra de la Restauración ... Op. cit., p. 120 
el horizonte de la hoja aromática. Los precios del tabaco oscilaban en el período correspondiente a los años 1838-1843, de un máximo de 189 marcos hasta un mínimo de 107. Esta coyuntura se presenta después de una larga fase de precios bastante altos en 1838 hasta 1841 , de un promedio de 170 marcos, a una abrupta caída a 111 marcos en 1843 .

Tabla N. ${ }^{\circ} 6$

Importación de Tabaco Santo Domingo-Hamburgo

Quintales métricos a $100 \mathrm{Kg} /$ Marcos

Años 1837-1843

\begin{tabular}{|r|r|r|r|}
\hline Años & $\mathbf{Q} / \mathbf{m}$ & M/p. 100 kg & Marcos/Total \\
\hline 1837 & 817 & 125.12 & 120,613 \\
1838 & 3,686 & 194.36 & 716,411 \\
1839 & 3,917 & 162.78 & 637,609 \\
1840 & 2,420 & 174.92 & 423,306 \\
1841 & 5,806 & 170.06 & 987,368 \\
1842 & 4,498 & 119.04 & 535,441 \\
1843 & 4,675 & 110.53 & 516,727 \\
\hline Total & & $\mathbf{3 , 9 3 7 , 4 7 5}$ \\
\hline
\end{tabular}

Analizando el volumen agregado o los valores exportados, la situación no es tan triste. El tabaco dominicano mantenía su alto nivel de exportación en la que la importación de los puertos alemanes Hamburgo y Bremen del año 1840 reportaban que los alemanes pagaban 286,862 marcos-banco y aumentaron su compra a 962,037 marco-banco en 1843. Una subida de 235\% (sic).

¿Qué pasó entonces? Esta caída abrupta de los precios del tabaco ¿provocó una crisis en el ánimo público que se transformó en un descontento general que llevó a las masas de la pobla- 
Tabla N. ${ }^{\circ} 7$

Importación de Tabaco

Santo Domingo-Hamburgo y Bremen

Valores/Marco banco en \%

\begin{tabular}{|c|r|r|r|r|}
\hline Años & Hamburgo & Bremen & Total & En \% \\
\hline 1840 & 109,134 & 177,728 & 286,862 & 100 \\
1841 & 357,485 & 426,418 & 783,903 & 273 \\
1842 & 329,736 & 330,346 & 660,082 & 230 \\
1833 & $\mid 618,716$ & 343,307 & 962,037 & 335 \\
\hline
\end{tabular}

\section{Fuentes:}

Hamburgo: Soetebeer. Op. cit. Tomo II, p. 159

Bremen: Comercio de Bremen en diferentes años

ción bajo sus grupos dirigenciales (por lo general, gente de negocio y en su mayoría comerciantes), al movimiento de la independencia?

La caída de precio del producto principal de la región, el tabaco, bajó las ganancias de los actores en el juego de manera muy diferente. No bajaron las entradas de los peones en la cosecha, en los ranchos, de los ayudantes de los recueros, de los trabajadores en los almacenes. Los ingresos del sector del transporte, de los recueros y de las familias que producían y movían los serones de los campesinos a los almacenes, no dependían del precio a bordo del barco en Puerto Plata.

El movimiento de la ciudad con sus múltiples funciones de servicio dependía más de manera indirecta de los buenos precios. ¿Cuál de los tres grupos perdió más en el año 1843, los consignatorios en Hamburgo y Bremen, los de Puerto Plata o los comerciantes de Santiago? Tal vez fueron capaces de presionar más a los productores y a los diferentes intermediarios. No tenemos ninguna información sobre los años 1842 y 1843 . Lo que sabemos es que las oscilaciones cerca del campesino eran muy 
reducidas en comparación con el consignatario que actuaba a nivel internacional. En buenos años el exportador ganaba una fortuna, en malos años podría sufrir grandes pérdidas, que con frecuencia lo conducían a la quiebra o a un estado de deudas que nunca lo dejaba recuperar su viejo estatus de comerciante.

Si el precio bajaba un peso $F$ por quintal, el cosechador, con su producción promedio de 5 quintales, perdía cinco pesos en esta cosecha. El precio es la medida de la calidad de la hoja de primera que servía como capa para el cigarro en Europa. El procesamiento en el rancho, el empaque sobre el hombro de la mula entre Santiago y Puerto Plata podría bajar la calidad a un nivel inferior. La comercialización de la hoja aromática, sobre todo para las calidades primeras del cigarro, estaba caracterizada siempre por una especulación alta. La alta calidad y el crecimiento de la exportación un $139.9 \%$ hacia el puerto de Hamburgo en el año 1840-1841 hizo crecer la esperanzas hasta el cielo y elevó las entradas en moneda fuerte para el Cibao un $133.2 \%$.

Era grande el optimismo reinante en el Cibao, después de cinco años consecutivos con precios tan altos que nunca se habían visto en la región. Ni los viejos se recordaban de algo mejor.

Entonces llegó el año fatal 1842 en el que el precio cayó casi un $30 \%$ y las entradas de la región productiva en dinero de oro y plata en un $45.6 \%$. (sic). Este año fastidioso fue seguido por un año de bajo precio, en el que perdió otra vez un 7.2\%. Este proceso aumentó las tendencias inflacionarias y subió la presión al consumidor. Así se muestran los elementos económicos de nuestra curva-ERO.

¿Por qué bajó el precio de forma repentina? En primer lugar hubo un descenso coyuntural en los mercados europeos. El informe de la bolsa de mercancías de Hamburgo para el año 1842 expone: "El comercio estaba muy animado, pero estas ventas se realizaban solamente por la baja provocada en los precios. (...) 
los envíos de café subieron en 2 millones de libras, pero los precios bajaron un $30 \% .{ }^{49}$ La importación de tabaco de Virginia y Kentucky casi se dobló, pero hubo una reducción en los precios. Al contrario se aumentó la demanda de las variedades de Havana y Santo Domingo pagando unos precios ligeramente elevados en comparación con el año anterior (1841). ${ }^{50}$

Gran parte del tabaco importado fue comprado en los países productores por orden de grandes casas comerciales o fábricas de tabaco y cigarro.

El ligero descenso coyuntural del año 1842 no nos explica la caída de precio de casi un 50\% en el mercado de Hamburgo. Lo que bajó enormemente fue la calidad del tabaco en las cosechas de los años 1842-1843.

El año 1842 fue fatal y parecía para los contemporáneos un Apocalipsis por

"... los ruinosos efectos del espantoso terremoto que se sintió de un extremo a otro de la isla del día 7 de mayo $1842[\ldots]$ reduciendo a escombros las poblaciones de Santiago, La Vega, Cab Haitiano, Portau Paix, El Mole, Sanit Marc y Gonaives; desmejoró los edificios más sólidos de la ciudad de Santo Domingo; y sumergió la punta de Manzanillo o Jicaco, en la costa del Norte. [...] En muchas partes se abrieron anchas y hondas grietas, y dos personas fueron tragadas hasta más de la mitad del cuerpo; los ríos presentaron también la más extraña revolución en el orden natural, retrocediendo de sus corrientes en el caso del estremecimiento más potente; en algunas partes se notó que saltaba el agua en borbollones como hirviendo. No hubo en toda la isla lugar que no experimentara daños más o menos considerables; pero llaman la atención aquellos pueblos que fueron arrasados por efecto del terremoto, invadidos por el mar, y consumidos por las llamas". $\$$

\footnotetext{
49 Tooke, Tn. y Newmarch, W. A history of Prices and of the State of the Circulation from 1793-1857. Usamos la versión alemana, traducido por Ascher, C. W. «Die Geschiche und die Bestimmung der Preise", Tomo I, Dresden, 1862, p. 565

${ }^{50}$ Tooke y Newmarch. A history ... Op. cit., p. 566

s1 Gabriel Garcia, José. Compendio ... Op. cit., Tomo II, p. 177
} 
La zona tabacalera con su centro comercial Santiago sufrió "... la inesperada calamidad de un desenfrenado pillage en los momentos mismos en que la tierra aún todavía removida, amedrentaba con fuertes y repetidos sacudimientos". ${ }^{52}$ Apareció el mismo día 7 de mayo un gran meteoro y siguieron muchos temblores entre grandes y pequeños durante casi dos meses.

"Estos temblores, tan repetidos, dejaron reducida la isla al estado mas lastimoso, porque el temor se apoderó de todos los corazones y el arrepentimiento de todas las conciencias ocasionando una paralización tan completa, que la miseria no se hizo esperar mucho tiempo". ${ }^{53}$

Este "fin del mundo" creó "... las condiciones más favorables para un cambio de gobierno". ${ }^{54}$

\section{Gráfica N ${ }^{\circ} 3$}

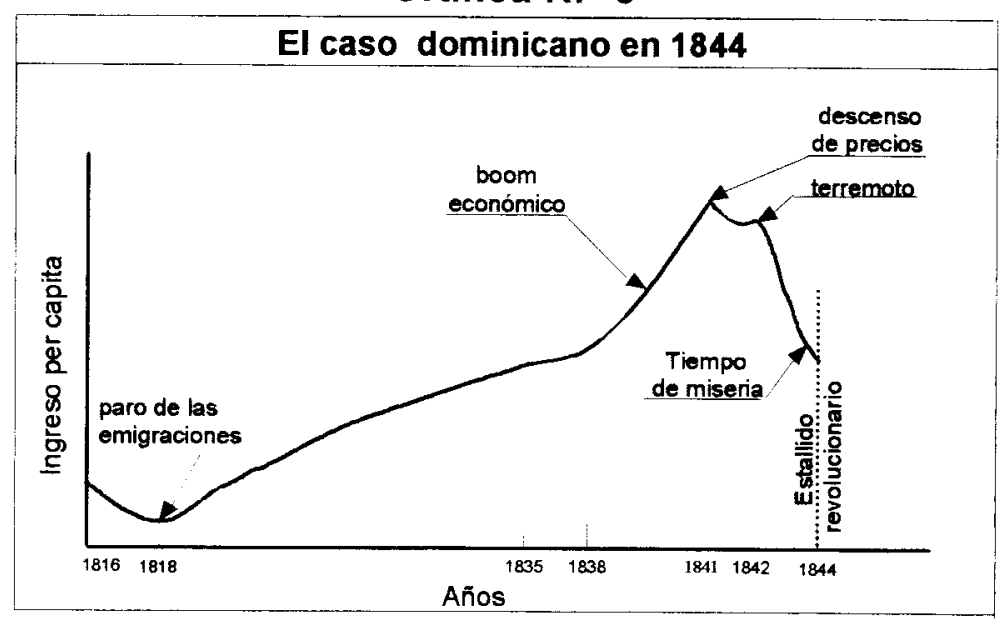

Nuestra curva-ERO está caracterizada a base de un período largo próspero desde el año 1818 hasta el año 1843 y más. Con la apertura de los puertos de la parte española para el comercio

\footnotetext{
\$: Gabriel García, José Compendio ... Op. cit.. Tomo II. p. 178

:Gabriel García, José Compendio ... Op. cit., Tomo II. p. 179

${ }^{54}$ Schoenrich, Otto. Santo Domingo ... Op. cit.. p. 36
} 
internacional y el paro de las emigraciones, comenzó un período largo de bienestar que se mantuvo durante 45 años, acompañado por una curva ascendente de crecimiento alto de la población, producción y exportación, obteniendo por esta vía elevados ingresos en divisas y su distribución interna igualitaria, produciendo así un período largo de un bienestar social relativamente creciente. Las capas más bajas de la población isleña recibieron mejores ingresos que la mayoría de la población europea de su época, que era una sociedad en pleno desarrollo hacia la Revolución Industrial.

La gran mayoría de la población española celebró la incorporación de la parte Este a la República de Haití, porque trajo la abolición de la esclavitud y la igualdad social y jurídica. La formulación del código rural haitiano dio, la primera vez en la historia, a las clases bajas trabajadoras, como peones, sirvientes, muchachos y aprendices derechos contractuales hacia sus patrones. Muchos campesinos liberaron sus tierras de los censos y cargas por las capillarias. Un período corto de boom económico, entre 1838 y 1841, hizo renacer las Esperanzas Reales Obtenidas "hasta el cielo". En este momento se presentó de golpe una crisis fuerte, apocalíptica, que llevó a las clases dirigentes y a las masas de la población a la Independencia en 1844.

\section{BIBLIOGRAFÍA}

1. Archambault, Pedro M. Historia de la Restauración, Santo Domingo, 1986. Primera edición. Paris, 1938, en. La Libraire Technique et Economique.

2. Baasch, Ernst. Historia de Hamburg, 1818 - 1918, Hamburgo, 1924 (Geschichte Hamburgs).

3. Baasch, Ernst. Historia económica de Holanda, Jena, 1927 (Hollandische Wirtschaftsgeschichte)

4. Boin, Jacqueline. Serulle Ramia, José. El proceso de desarrollo del capitalismo en la República Dominicana (1844), Santo Domingo, 1984, Tomo I

5. Bosch, Juan. Composición Social Dominicana, Historia e Interpretación, trigésima edición, Santo Domingo, 1983 
6. Bosch, Juan. Guerra de la Restauración, 1991, 7. Edit.

7. Brinton, Crane. The Anatomy of Revolution, New York, 1952

8. Cassá, Roberto. Historia Social y Económica de la República Dominicana, cuarta edición, Tomo II, Santo Domingo, 1983

9. Cohén, E. A. Human behavior in the Concentration Camp, New York, 1953

10. Davies, James C. "Toward a Theory of Revolution", en American Sociological Review, 27, (1962)

11. Delitsch, Otto. Oesteindia y los paises de la Antártica, Leipzing 1871 (Westindien und die Lander der Antarktis)

12. Engelisng, Rolf. Bremen como puerto de emigración, 1683-1880, Bremen, 1961, (Bremen als Auswandererhafen)

13. Franklin, James. The Present State of Haiti, London, 1828

14. Gabriel García, José. Compendio de la Historia de Santo Domingo, quinta edición, Santo Domingo, 1982, Tomo I, II, III

15. Guerra y Sánchez, Ramiro. Manual de Historia de Cuba desde su descubrimiento hasta 1868, 4a edición. La Habana, 1971

16. Guridi, Alejandro Angulo. "Santo Domingo y España", en: Rodríguez Demorizi, Emilio. Antecedentes de la Anexión a España, Ed. Montalvo, Santo Domingo.

17. Huntington, Samuel. "Paterns of Violence in World Politics", en Channing Patterns of Military Politics, editado por. S. Huntington, New York, 1962.

18. Jesús Domínguez, Jaime de. La Anexión de la República Dominicana a España, Tomo I, Santo Domingo, 1979

19. Jesús Domínguez, Jaime de. "Economia Política en la República Dominicana, años 1944-1861", Colección Historia y Sociedad N. 29, Santo Domingo, 1977

20. Jesús, Jaime de. Notas Económicas y Políticas Dominicanas sobre el Período julio 1865-julio 1886, tomo I

21. Jimenes Grullón, Juan I. Sociología Politica Dominicana 1844-1966, Volumen I (1844-1898), 4ª Edición, Sto. Dgo. 1982

22. Lasswell, Harold y Kaplan, Abraham. Power and Society, New Haven, Conneticut,. 1950

23. Lepelletier de Saint Remy, R. Santo Domingo Estudio y solución mueva de la cuestión haitiana, Tomo II, Santo Domingo, 1978, el original en francés fue publicado en París en el año 1846 
24. Lieuwen, Edwin. Arms and Politics in Latin America, New York, 1960

25. Lluberes, Antonio. José del Castillo, Ramón Alburquerque. Tabaco, Azúcar y Mineria, Santo Domingo, 1984, "La Crisis del Tabaco Cibaeño, 1879 - 1930"

26. López Morillo, Adriano. Memorias sobre la Segunda Reincorporación de Santo Domingo a España, Tomo II, Santo Domingo, 1983

27. Luperón, Gregorio. Notas Autobiográficas y apuntes históricos, Santo Domingo, 1974, Tomo I, p. 148. Este primer tomo se publicó en el año 1895 en Ponce, Puerto Rico

28. Marte, Roberto. Cubay la República Dominicana Transición Económica en el Caribe del Siglo XIX, Santo Domingo, 1988

29. Marte, Roberto. Estadísticas y Documentos Históricos sobre Santo Domingo (1805-1890), Santo Domingo, 1984

30. Marx, Engels. Obras completas Tomo 6, Berlín-Este, 1961

31. Martínez, Rufino. Diccionario Biográfico-Histórico, 1821-1930, Santo Domingo, 1971.

32. Moya Pons, Frank. La Dominación Haitiana 1822-1844, $3^{3}$ Edición, Santiago, 1978

33. Moya Pons, Frank. El pasado dominicano, Santo Domingo, 1986, $1^{\text {a }}$ edición, 1986, "La Economía Dominicana en la Primera República"

34. Rodríguez Demorizi, Emilio (Edit.). Papeles de Pedro F Bonó, para la historia de las ideas politicas en Santo Domingo, Segunda Edición, Barcelona, 1980, en. Academia de la Historia, Vol. XVII

35. Rosenau, James N. "Internal War as an International Event", en: Internacionl Aspects of Civil Strife, editado por. James N. Rosenau, Princeton, N.J. 1964

36. Schoenrich, Otto. Santo Domingo un País con Futuro, Santo Domingo, 1977. El origina es en inglés, publicado en 1918 en New York

37. Siebek, Hermann. Aristóteles, Stuttgart, 1902, (alemán)

38. Soetbeer, A.. Aportaciones para la Estadistica de Precios, Hamburgo, 1859, Tomo I y II, Sign.:O III 7, Biblioteca de la Cámara de Comercio de Bremen, (Bibliothek der Handelskammer Bremen)

39. Tanter, Reymond. Midlarsky, Manus. "A Theory of Revolution", en Journal of Conflict Resolution, II (1967). Editado por la University of Michigan 
40. Tooke, The y Newmarch, W. A History of Princes and of the State of the Circulation from $1793-1857$, usamos la versión alemana, traducida por Ascher, Dr. C. W. (Die Geschichte und die Bestimmung der Preise) Tomo I, Tomo II, (anexo XXXIII. Comercio y precios de la bolsa de Hamburgo 1848-58), Dresden, 1859, sign. O III 5, Biblioteca de la Cámara de Comercio de Bremen (Bibliothek der Handelskammer Bremen)

41. Tocqueville, Alexis de. El estado viejo y la Revolución (Der alte Staat und die Revolution) aleman, Colect. 232, Biersfelden bei basel

42. Tsouyopoulus, Nelly. Grassi, Enesto (Edit). Aristóteles. Politica, libro cuarto, cap. 11 ; München, 1965,

\section{Fuentes Estadísticas:}

1. Anuario de Estadistica Bremen. (Jahrbuch L. bremiscbre Stalistik), 1860 - 1890, (Staatsarchiv der Freien Hansestadt Bremen)

2. Comercio de Bremen, diferentes años, desde 1837, editado por la Oficina Estadística, Bremen, 1837-81,(Staatsarchiv der Freien Hansestadt Bremen)

3. Periódico de Tabaco alemán, No. 14, 8 de abril 1870,3. Año, los datos de la importación de tabaco en el puerto de Hamburgo (Deutsche Tabakzeitung)

4. Estadística de importación de tabaco en Hamburgo (véase) hasta 1850. Libro de Mercancias, dif. Años, Hamburg, en Zoll y Akzisewesen, C. A. 1. Tomo 17 ss. Y para 1851 arriba, Tomo 8, No. 10 Microfilmado KA 310 F, STA-Hambur (Hamburger Staasarchiv)

5. Zoll-u- Akzisewesen, Band VIII, Nr. 10, (Staatsarchiv Hamburg)

\section{Documentos de los archivos notariales}

(PP-Puerto Plata, $M=$ Moca, IV - La Vega, IIII = Hamburgo)

1. AN-PP, No.16, 9 de marzo 1858, Hipoteca casa. Documentos de los archivos dominicanos

2. Heneken, Teodoro Staley: en carta a Ribero, legajo No.7, de la Anexión a España, AGND Documentos de los archivos elemanes

3. Informe consular Puerto Plata, Federico Finke, 31 de diciembre 1857 STAOL, Best. 31-13-16 No.55 (Staatsarchiv Oldenburg) 
4. Informe consular, año 1857 de Carlos Neumann, Cónsul de Prusia y Austria en Puerto Plata, Puerto Plata, 5 de enero 1858, GSTAMerseburg, Ministerio del Exterior, 2.4.1. Abt. II, No. 5258, hoja 5363, (Geheimes Staatsarchiv Merseburg)

5. Informe consular, año 1858 de Carlos Neumann, Cónsul de Prusia y Austria en Puerto Plata, Puerto Plata, 15 de febrero 1859, GSTAMerseburg, Staatsarchiv Merseburg)

6. Informe consular, año 1858 de Federico Finke, Cónsul de Oldenburgo en Puerto Plata, Puerto Plata, 31 de diciembre 1858, Sign. Best. 31 - 15 44, Nr. 110 , Fasc, STAOLD, (Staatsarchiv Oldenburg)

7. Informe consular, año 1858 de Luis Sander, cónsul de Hamburgo en Puerto plata, Puerto Plata, 31 de diciembre 1858, Sign. H 516, 59, Biblioteca de la Cámara de Comercio de Hamburgo, (Bibliothek der Handelskammer in Hamburg

8. Informe consular, año 1861 de Carlos Neumann, Cónsul prusiano en Puerto Plata, 31 de diciembre 1861, Puerto Plata GSTA-Merseburg, 2.4.1. Abt. II, No.5258, hoja 79-80 (Geheimes Staatsarchiv Merseburg)

9. Informe consular, año 1862 de Carlos Neumann, Cónsul de Prusia y Austria en Puerto Plata, Puerto Plata, 1 de enero 1863, Puerto Plata, GSTA-Merseburg, 2.4.1. Abt. II, No.5258, hoja 84-95 (Geheimes Staatsarchiv Merseburg)

10. Informe consular, año 1862, de Federico Finke, Puerto Plata, 25 de marzo 1863, en informes consulares de Oldenburgo, Sign. STA-OLD, Best. 31-15-11 No. 161 B (Staatsarchiv Oldenburg)

11. Warenpreiscouranten, Bremen, 1842 - 1860 Sign 2-Ss.21.b. Tomo 1, STA-Bremen (Staatsarchiv Bremen)

12. Warenpreiscouranten, Bremen, $1842-1860$, Sign. 2-Ss. 1A21.b Tomo 3, STA-Bremen (Staatsarchiv Bremen)

\section{Periódicos alemanes del siglo xix}

1. Hoja de Comercio Bremes (Bremer Handelsblatt), No. 49, 11 de septiembre 1852

2. Hoja de Comercio Bremes (Bremer Handelsblatt), No. 58, 13 de noviembre 1852

3. Hoja de Comercio Bremes (Bremer Handelsblatt), No. 59, 20 de noviembre 1852 
4. Hoja de Comercio Bremes (Bremer Hnadelsblatt), No. 278, 7 de febrero 1857

5. Hoja de Comercio Bremes (Bremer Handelsblatt), No. 279, 14 de febrero 1857

6. Hoja de Comercio Bremes (Bremer Handelsblatt), No. 331, 13 de febrero

7. Hoja de Comercio Bremes (Bremer Handelsblatt), No. 381, 29 de enero 1859 